\title{
TRANSFORMATIONS OF CERTAIN GENERALIZED KAMPÉ DE FÉRIET FUNCTIONS II
}

\author{
HAROLD EXTON \\ "Nyuggel", Lunabister, Dunrossness \\ Shetland ZE2 9JH, United Kingdom
}

(Received May, 1996; Revised October, 1996)

The development of identities of multivariable hypergeometric functions is further extended based upon the methods of the previous study (H. Exton, J. Phys. A29 (1996), 357-363) these functions occur in various applications in the fields of physics and quantum chemistry.

Key words: Hypergeometric Multivariable.

AMS subject classifications: $33 \mathrm{C} 50,33 \mathrm{C} 45,33 \mathrm{C} 90$.

\section{Introduction}

This study is a sequel to the work carried out by Exton [3] in which transformations of the Kampé de Fériet functions of several variables, Karlsson [4], are obtained. This type of function has applications in mathematical physics and has previously been discussed by Niukkanen [5] and [6] and Srivastava [8-11] for example. The reader should consult these references for a detailed description of the applications of the type of function under consideration. It is denoted by

$$
\begin{gathered}
F^{A: C: B: D}\left[\begin{array}{c}
a_{1}, a_{2}, \ldots, a_{A} ; b_{1,2}, \ldots, b_{1, B} ; b_{2,1}, \ldots, b_{2, B} ; \ldots ; b_{n, 1}, \ldots, b_{n, B} ; \\
c_{1}, c_{2}, \ldots, c_{C}, d_{1,2}, \ldots, d_{1, D} ; d_{2,1}, \ldots, d_{2, D} ; \ldots ; d_{n .1}, \ldots, d_{n . D} ;
\end{array}\right] \\
=F^{A: C:^{b} D}\left[\begin{array}{l}
(a):\left(b_{1}\right) ; \ldots ;\left(b_{n}\right) ; \\
(c):\left(d_{1}\right) ; \ldots ;\left(d_{n}\right) ;
\end{array}\right]
\end{gathered}
$$

with series representation

$$
\sum \frac{((a), \Sigma m)\left(\left(b_{1}\right),{ }_{1}\right) \ldots\left(\left(b_{n}\right), m_{n}\right) x_{1}^{m} 1 \ldots x_{n}^{m} n}{((c), \Sigma m)\left(\left(d_{1}\right), m_{1}\right) \ldots\left(\left(d_{n}\right), m_{n}\right) m_{1} ! \ldots m_{n} !}
$$

in which the indices of summation run over all of the non-negative integers. The symbol $(a)$ is a convenient contraction for the sequence of parameters $a_{1}, a_{2}, a_{A}$ and the Pochhammer symbol $(a, n)$, as usual, is given by 


$$
(a, n)=\Gamma(a+n) / \Gamma(a)=a(a+1) \ldots(a+n-1) ;(a, 0)=1 .
$$

Also, $\Sigma m=m_{1}+\ldots+m_{n}$, etc., and any values of parameters for which any results do not make sense are tacitly excluded. The above notation is discussed at greater length in Exton [2] and [3], for example.

The following general result was presented in Exton [3] and is stated here for completeness:

$$
\begin{aligned}
& \sum \frac{((a), \Sigma m)((d), \Sigma m)\left(\left(c_{1}\right), m_{1}\right)\left(\left(q_{1}\right), m_{1}\right)\left(\left(z_{1}\right), 2 m_{1}\right)}{((h), \Sigma m)((g), \Sigma m)\left(\left(p_{1}\right), m_{1}\right)\left(\left(b_{1}\right), m_{1}\right)\left(\left(k_{1}\right) \cdot 2 m_{1}\right)} \\
& \ldots \frac{\left.\left(\left(c_{n}\right), m_{n}\right)\left(\left(q_{n}\right), m_{n}\right)\left(\left(z_{n}\right), m_{n}\right)\left(x_{1}, y_{1}\right)^{m_{1}} \ldots x_{n} y_{n}\right)^{m_{n}}}{\left(\left(p_{n}\right), m_{n}\right)\left(\left(b_{n}\right), m_{n}\right)\left(\left(k_{n}\right), m_{n}\right) m_{1} ! \ldots m_{n} !} \\
& \times F^{D:} G:{ }^{Q+Z} B+K
\end{aligned}
$$

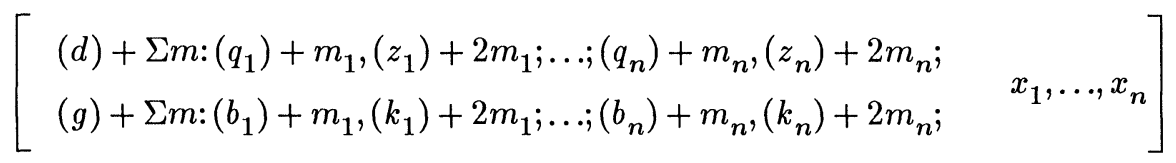

$$
\begin{aligned}
& =\frac{((d), \Sigma m)\left(\left(q_{1}\right), m_{1}\right)\left(\left(z_{1}\right), m_{1}\right) \ldots\left(\left(q_{n}\right), m_{n}\right)\left(\left(z_{n}\right), m_{n}\right) x_{1}^{m_{1}} \ldots x_{n}^{m_{n}}}{((g), \Sigma m)\left(\left(b_{1}\right), m_{1}\right)\left(\left(k_{1}\right), m_{1}\right) \ldots\left(\left(b_{n}, m_{n}\right)\left(\left(k_{n}\right), m_{n}\right) m_{1} ! \ldots m_{n} !\right.} \\
& \times F^{A:} H:{ }^{C+Z+1} P+K \\
& {\left[\begin{array}{c}
(a):\left(c_{1}\right),\left(z_{1}\right)+m_{1},-m_{1} ; \ldots ;\left(c_{n}\right),\left(z_{n}\right)+m_{n},-m_{n} ; \\
(h):\left(p_{1}\right),\left(k_{1}\right)+m_{1} ; \ldots ;\left(p_{n}\right),\left(k_{n}\right)+m_{n} ;
\end{array}\right]}
\end{aligned}
$$

The procedure now used consists of expressing the inner generalized Kampé de Fériet functions in compact form, thus obtaining the transformations sought. Any questions of convergence must be dealt with in each individual case as appropriate. The reader is referred to Exton [3] for more details of the above preliminaries.

The following formulae are required in the subsequent analysis:

$$
\begin{aligned}
& { }_{1} F_{0}[a ;-; x]=(1-x)^{-a}, \quad(\text { the binomial theorem }) \\
& { }_{2} F_{1}[a, b ; c ; x]=(1-x)^{c-a-b}{ }_{2} F_{1}[c-a, c-b ; c ; x], \\
& F^{A: C:{ }^{1} 0}\left[\begin{array}{ll}
(a): b_{1} ;,, ; b_{n} ; & \\
(c):-;,, ;-; &
\end{array}\right]={ }_{A+1} F_{A}[(a), \Sigma b ;(c) ; x], \\
& F^{0}: 0:{ }^{B} D\left[\begin{array}{l}
-:\left(b_{1}\right) ;,, ;\left(b_{n}\right) ; \\
-:\left(d_{1}\right) ;,, ;\left(d_{n}\right) ;
\end{array} \quad x_{1}, \ldots, x_{n}\right]=B^{F} D\left[\left(b_{1}\right) ;\left(d_{1}\right) ; x_{1}\right] \ldots B^{F} D\left[\left(b_{n}\right) ;\left(d_{n}\right) ; x_{n}\right],
\end{aligned}
$$




$$
\begin{gathered}
{ }_{2} F_{1}[a,-n ; c ; 1]=(c-a, n) /(c, n), \quad \text { (Vandermonde's theorem) } \\
{ }_{3} F_{2}[a, b,-n ; c, a+b-c-n+1 ; 1]=[(c-a, n)(c-b, n)] /[(c, n)(c-a-b, n)], \\
\quad \text { Saalschütz's theorem) } \\
{ }_{3} F_{2}[a, b, c ; 1 / 2+a / 2+b / 2,2 c ; 1] \\
=[\Gamma(1 / 2) \Gamma(c+1 / 2) \Gamma(1 / 2+a / 2+b / 2) \Gamma(1 / 2-a / 2-b / 2+c)] / \\
{[\Gamma(1 / 2+a / 2) \Gamma(1 / 2+b / 2) \Gamma(1 / 2-a / 2+c) \Gamma(1 / 2-b / 2+c)]} \\
\quad \text { Watson's theorem) }
\end{gathered}
$$

and

$$
{ }_{4} F_{3}[a / 2, a / 2+1 / 2, b+n,-n ; b / 2, b / 2+1 / 2,1+a ; 1]=(b-a, n) /(b, n) .
$$

The results (1.4) and (1.8) through (1.11) are to be found in Slater [7], Appendix III. For the formulae (1.6) and (1.7), see Srivastava and Karlsson [12, page 39] and for (1.5), see Erdélyi [1, page 105]. In the following analysis, any expressions which are trivial or which reduce immediately to transformations of one dimension are omitted. In order to save space, much of the detail of the working is left out, and the reader should consult Exton [3] if further clarification is desired.

\section{A Transformation Deduced from the Binomial Theorem}

In the general transformation (1.3), put $D=G=Q=1, Z=B=K=0$ and let $x_{1}=\ldots=x_{n}=x$ and $g=\Sigma q$. As a simple consequence of (1.6), we see that the inner Kampé de Fériet function on the left takes the form

$$
\begin{aligned}
& F^{1: 1:{ }^{1} 0}\left[\begin{array}{c}
d+\Sigma m: q_{1}+m_{1} ; \ldots ; q_{n}+m_{n} ; \\
\Sigma q+\Sigma m:-; \ldots ;-;
\end{array}\right] \\
& ={ }_{2} F_{1}[d+\Sigma m, \Sigma q+\Sigma m ; \Sigma q+\Sigma m ; x]=(1-x)^{-d-\Sigma m} .
\end{aligned}
$$

The expression (1.3) then becomes

$$
\begin{aligned}
& (1-x)^{-d} \sum \frac{((a), \Sigma m)(d, \Sigma m)\left(q_{1}, m_{1}\right) \ldots\left(q_{n}, m_{n}\right)}{((h), \Sigma m)(\Sigma q, \Sigma m)} \\
& \times \frac{\left.\left(\left(c_{1}\right), m_{1}\right) \ldots\left(c_{n}\right), m_{n}\right)\left[x y_{1} /(1-x)\right]^{m_{1}} 1 \ldots\left[x y_{n} /(1-x)\right]^{m_{n}}}{\left(\left(p_{1}\right), m_{1}\right) \ldots\left(\left(p_{n}\right), m_{n} ! \ldots m_{n} !\right.} \\
& =\sum \frac{(d, \Sigma m)\left(q_{1}, m_{1}\right) \ldots\left(q_{n}, m_{n}\right) x^{\Sigma m}}{(\Sigma q, \Sigma m) m_{1} ! \ldots m_{n} !} \\
& \times F^{A: H:}{ }^{C+1} P\left[\begin{array}{cc}
(a):\left(c_{1}\right),-m_{1} ; \ldots ;\left(c_{n}\right),-m_{n} ; & -y_{1}, \ldots,-y_{n} \\
(h):\left(p_{1}\right) ; \ldots ;\left(p_{n}\right) ; &
\end{array}\right]
\end{aligned}
$$


If $A=H=0$, the inner generalized Kampé de Fériet function on the right may be so specialized as to be summable by (1.8). After some algebra, we then have the result

$$
\begin{gathered}
(1-x)^{-d} F_{1:}^{1:} 2_{1}\left[\begin{array}{c}
d: c_{1}, q_{1} ; \ldots ; c_{n}, q_{n} ; \\
\Sigma q: p_{1} ; \ldots ; p_{n} ;
\end{array}\right] \\
\left.=F^{1:} 2_{1:}\left[\begin{array}{c}
d: p_{1}-c_{1}, q_{1} ; \ldots ; p_{n}-c_{n}, q_{n} ; \\
\Sigma q: p_{1} ; \ldots ; p_{n} ;
\end{array}\right] ., \quad x, \ldots, x\right]
\end{gathered}
$$

\section{Three-term Transformation Deduced from (1.5)}

The inner series on the left of (1.3) can be expressed in closed form by means of (1.6) and (1.5) after suitably adjusting the parameters and variables. If $D=Z=G=1$, $A=G=Q=B=K=0, x_{1}=\ldots=x_{n}=x$ and $g=d-1$, this inner series becomes

$$
\begin{aligned}
&\left.F^{1:}{ }_{1: 1}\left[\begin{array}{c}
d+\Sigma z: z_{1}+2 m_{1} ; \ldots ; z_{n}+2 m_{n} ; \\
d-1+\Sigma z:-; \ldots ;-;
\end{array}\right], x\right]\left(\begin{array}{c} 
\\
=
\end{array}{ }_{2} F_{1}[d+\Sigma m, \Sigma z+2 \Sigma m ; d-1+\Sigma m ; x]\right. \\
&=(1-x)^{-1-\Sigma z-2 \Sigma m}{ }_{2} F_{1}[-1, d-\Sigma z-1-\Sigma m ; d-1+\Sigma m ; x] \\
&=(1-x)^{-1-\Sigma z-2 \Sigma m}[1+(\Sigma z-d+1+\Sigma m) /(d-1+\Sigma m) x] .
\end{aligned}
$$

Hence, (1.3) takes the form

$$
\begin{aligned}
& (1-x)^{-1-\Sigma z} F^{1:}{ }^{C+2} P \\
& {\left[\begin{array}{lll}
d:\left(c_{1}\right), \frac{z_{1}}{2}, \frac{z_{1}}{2}+\frac{1}{2} ; \ldots ;\left(c_{n}\right), \frac{z_{n}}{2}, \frac{z_{n}}{2}+\frac{1}{2} ; & \frac{4 x y_{1}}{(1-x)^{2}}, \ldots, \frac{4 x y_{n}}{(1-x)^{2}}
\end{array}\right]} \\
& -x(1-x)^{-1-\Sigma z}(d-\Sigma z-1) /(d-1) \\
& \times F^{1:} 1:{ }^{C+2} P \\
& \left.\begin{array}{ccc}
\Sigma z-d+2:\left(c_{1}\right), \frac{z_{1}}{2}, \frac{z_{1}}{2}+\frac{1}{2} ; \ldots ;\left(c_{n}\right), \frac{z_{n}}{2}, \frac{z_{n}}{2}+\frac{1}{2} ; & \frac{4 x y_{1}}{(1-x)^{2}}, \ldots, \frac{4 x y_{n}}{(1-x)^{2}} \\
\Sigma z-d+1: \quad\left(p_{1}\right) ; \ldots ; \quad\left(p_{n}\right) \quad ; &
\end{array}\right] \\
& =\sum \frac{(d, \Sigma m)\left(z_{1}, m_{1}\right) \ldots\left(z_{n}, m_{1}\right) x^{\Sigma m}}{(d-1, \Sigma m) m_{1} ! \ldots m_{n} !}
\end{aligned}
$$




$$
\times_{C+2} F_{p}\left[\left(c_{1}, z_{1}+m_{1},-m_{1} ;\left(p_{1}\right) ;-y_{1}\right] \ldots C+{ }_{2} F_{P}\left[\left(c_{n}\right), z_{n}+m_{n},-m_{n} ;\left(p_{n}\right) ;-y_{n}\right] .\right.
$$

The formulae (1.8) through (1.11) can then be used to sum the inner hypergeometric functions on the right of (3.2). In order to save space, the details of any working out are omitted, and the reader should see Exton [3]. The following results ensue, respectively:

$$
\begin{aligned}
& F^{1:} 1:{ }^{2} 1\left[\begin{array}{rlll}
d: \frac{z_{1}}{2}, \frac{z_{1}}{2}+\frac{1}{2} ; \ldots ; \frac{z}{2}, \frac{z}{2}+\frac{1}{2} ; & \\
d-1: & p_{1} \quad ; \ldots ; p_{n} \quad ; & \frac{-4 x}{(1-x)^{2}}, \ldots, \frac{-4 x}{(1-x)^{2}}
\end{array}\right] \\
& -x(d-\Sigma z-1) /(d-1) F^{1:} 1:^{2} 2 \\
& {\left[\begin{array}{llll}
\Sigma z-d+2: \frac{z_{1}}{2}, \frac{z_{1}}{2}+\frac{1}{2} ; \ldots ; \frac{z}{2}, \frac{z}{2}+\frac{z_{n}}{2} ; & \frac{-4 x}{(1-x)^{2}}, \ldots, \frac{-4 x}{(1-x)^{2}}
\end{array}\right]} \\
& =(1-x)^{1+\Sigma z} F^{1:} 1:^{2} 2
\end{aligned}
$$

$$
\left[\begin{array}{ccccc}
d: z_{1}, 1+z_{1}-p_{1} ; \ldots ; z_{n}, 1+z_{n}-p_{n} ; & -x, \ldots,-x \\
d-1: & p_{1} & ; \ldots ; & p_{n} & ;
\end{array}\right]
$$

$F^{1:} a:{ }^{3} 2$

$$
\begin{aligned}
& {\left[\begin{array}{cc}
d: c_{1}, \frac{z_{1}}{2}, \frac{z_{1}}{2}+\frac{1}{2} ; \ldots ; c_{n}, \frac{z_{n}}{2}, \frac{z_{n}}{2}+\frac{1}{2} ; & \frac{-4 x}{(1-x)^{2}}, \ldots, \frac{-4 x}{(1-x)^{2}}
\end{array}\right]} \\
& -x(d+\Sigma z-1) /(d-1) F^{1:} 1:^{3_{2}} \\
& {\left[\begin{array}{cc}
\Sigma z-d+2: c_{1}, \frac{z_{1}}{2}, \frac{z_{1}}{2}+\frac{1}{2} ; \ldots ; c_{n}, \frac{z_{n}}{2}, \frac{z_{n}}{2}+\frac{1}{2} ; & \frac{-4 x}{(1-x)^{2}}, \ldots, \frac{-4 x}{(1-x)^{2}} \\
\Sigma z-d+1: p_{1}, c_{1}+z_{1}-p_{1}+1 ; \ldots ; p_{n}, c_{1}+z_{n}-p_{n}+1 ; &
\end{array}\right]} \\
& =(1-x)^{1+\Sigma z} F^{1:} 1:^{3} 2 \\
& {\left[\begin{array}{c}
d: z_{1}, p_{1}-c_{1}, 1+z_{1}-p_{1} ; \ldots ; z_{n}, p_{n}-c_{n}, 1+z_{n}-p_{n} ; \quad-x, \ldots,-x \\
d-1: p_{1}, 1+z_{1}+c_{1}-p_{1} ; \ldots ; p_{n}, 1+z_{n}+c_{n}-p_{n} ;
\end{array}\right]}
\end{aligned}
$$

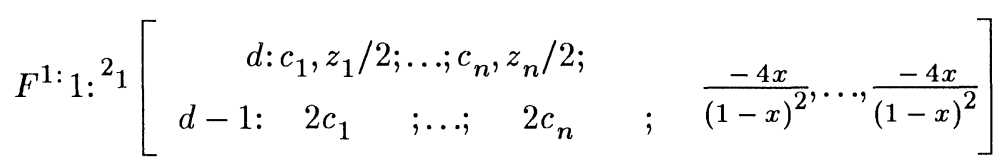




$$
\begin{aligned}
& -x(d-\Sigma z-1) /(d-1) F^{1:} 1:^{2} 1 \\
& {\left[\begin{array}{llll}
\Sigma z-d+2: c_{1}, z_{1} / 2, ; \ldots ; c_{n}, z_{n} / 2, ; & \frac{-4 x}{(1-x)^{2}}, \ldots, \frac{-4 x}{(1-x)^{2}} \\
\Sigma z-d+1: & 2 c_{1} & ; \ldots ; & 2 c_{n}
\end{array}\right]} \\
& =(1-x)^{1+\Sigma z} F^{1:}{ }_{1:}{ }^{2} \\
& {\left[\begin{array}{cccc}
d / 2+1 / 2: z_{1} / 2, z_{1} / 2+1 / 2-c_{1} ; \ldots ; z_{n} / 2, z_{n} / 2+1 / 2-c_{n} ; & x^{2}, \ldots, x^{2} \\
d / 2-1 / 2: & c_{1}+1 / 2 & ; \ldots ; & c_{n}+1 / 2
\end{array}\right]}
\end{aligned}
$$

and

$F^{1:}:^{2}{ }^{1}$

$$
\begin{aligned}
& {\left[\begin{array}{cccc}
d: a_{1} / 2, a_{1} / 2+1 / 2 ; \ldots ; a_{n} / 2, a_{n} / 2+1 / 2 ; & \frac{-4 x}{(1-x)^{2}}, \ldots, \frac{-4 x}{(1-x)^{2}} \\
d-1: & 1+a_{1} \quad ; \ldots ; 1+a_{n} \quad ; &
\end{array}\right]} \\
& -x(d-\Sigma z-1) /(d-1) F^{1:} 1:^{2} 1 \\
& {\left[\begin{array}{rrrr}
\Sigma z-d+2: a_{1} / 2, a_{1} / 2+1 / 2 ; \ldots ; a_{n}, a_{n} / 2+1 / 2 ; & \frac{-4 x}{(1-x)^{2}}, \ldots, \frac{-4 x}{(1-x)^{2}} \\
\Sigma z-d+1: & 1+a_{1} \quad ; \ldots ; & 1+a_{n} \quad ; &
\end{array}\right]}
\end{aligned}
$$

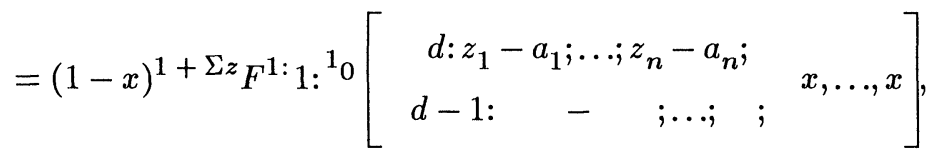

The right-hand side member of (3.6) can be simplified by appealing to (1.6) and (1.5). Hence,

$$
\begin{aligned}
& F^{1:} 1:{ }^{2} 1\left[\begin{array}{rrr}
d: a_{1} / 2, a_{1} / 2+1 / 2 ; \ldots ; a_{n}, a_{n} / 2, a_{n} / 2+1 / 2 ; \\
d-1: 1+a_{1} \quad ; \ldots ; & 1+a_{n} \quad ; \quad \frac{-4 x}{(1-x)^{2}}, \ldots, \frac{-4 x}{(1-x)^{2}}
\end{array}\right] \\
& -x(d+\Sigma z-1) /(d-1) F^{1:} 1:{ }^{2}
\end{aligned}
$$




$$
\begin{aligned}
& \Sigma z-d+2: a_{1} / 2, a_{1} / 2+1 / 2 ; \ldots ; a_{n} / 2, a_{n} / 2+1 / 2 \\
& \Sigma z-d+1: 1+a_{1} \quad ; \ldots ; 1+a_{n} ; \\
& \left.\frac{-4 x}{(1-x)^{2}}, \ldots, \frac{-4 x}{(1-x)^{2}}\right] \\
& =(1-x)^{\Sigma a}[1-(d-1-\Sigma z+\sigma a) /(d-1) x]
\end{aligned}
$$

\section{A Transformation of Higher Order}

This study is concluded by deducing a transformation of general order in which one of the variables is restricted. As such, this type of expression does not possess a single-variable analog.

In the general result (1.3), put $Q=Z=B=K=0$ and let $x_{n}=-x_{1}-\ldots$ $-x_{n-1}$. Utilizing an idea employed by Exton [2], we see that, as a consequence of (1.6), the inner Kampé de Fériet function on the left reduces to unity. Hence

$$
\begin{aligned}
& \sum \frac{((a), \Sigma m)((d), \Sigma m)\left(\left(c_{1}\right), m_{1}\right) \ldots\left(\left(c_{n}\right), m_{n}\right)\left(x_{1} y_{1}\right)^{m_{1}} \ldots\left(x_{n-1}, y_{n-1}\right)^{m_{n-1}}}{((h), \Sigma m)((g), \Sigma m)\left(\left(p_{1}\right), m_{1}\right) \ldots\left(\left(p_{n}\right), m_{n}\right) m_{1} ! \ldots m_{n} !} \\
& \times\left(-y_{n}\left[x_{1}+\ldots+x_{n-1}\right]\right)^{m} n \\
& =\sum \frac{((d), \Sigma m) x_{1}^{m_{1}} \ldots x_{n-1}^{m_{n}-1}\left(-x_{1}-\ldots-x_{n-1}\right)^{m}}{((g), \Sigma m) m_{1} ! \ldots m_{n} !} \\
& \times F^{A: H:}{ }^{C+1} P\left[\begin{array}{c}
(a):\left(c_{1}\right),-m_{1} ; \ldots ;\left(c_{n}\right),-m_{n} ; \\
(h):\left(p_{1}\right) \quad ; \ldots ; \quad\left(p_{n}\right) ;
\end{array}\right]
\end{aligned}
$$

If $A=H=0, C=P=1$ and $y_{1}=\ldots=y_{n}=-1$, the inner Kampé de Fériet function can be summed by means of (1.8). It then follows that

$$
\begin{aligned}
& F^{D:} G:{ }^{1}\left[\begin{array}{l}
(d): c_{1} ; \ldots ; c_{n} ; \\
(g): p_{1} ; \ldots ; p_{n} ;
\end{array} \quad=x_{1}, \ldots,-x_{n-1}, x_{1}+\ldots+x_{n-1}\right] \\
& =F^{D:} G:{ }^{1} 1\left[\begin{array}{c}
(d): p-c_{1} ; \ldots ; p_{n}-c_{n} ; \\
(g): p_{1} ; \ldots ; p_{n} ;
\end{array} \quad=x_{1}, \ldots, x_{n-1},-x_{1}-\ldots-x_{n-1}\right] .
\end{aligned}
$$

It is clear that this short list of transformation is far from complete. 


\section{References}

[1] Erdélyi, A., Higher Transcendental Functions, McGraw-Hill, New York 1953.

[2] Exton, H., Generating relations for Tratnik's multivariable biorthogonal continuous Hahn polynomials, J. Math. Phys. 33 1992), 524-527.

[3] Exton, H., Transformations of certain generalized Kampé de Fériet functions, $J$. Phys. A29 (1996), 357-363.

[4] Karlsson, P.W., Reduction of certain generalized Kampé de Fériet functions, Math. Scand. 32 (1973), 265-268.

[5] Niukkanen, A.W., Generalized hypergeometric series ${ }^{N} F\left(x_{1}, \ldots, x_{n}\right)$ arising in physical and quantum chemical applications, J. Phys. A16 (1983), 1813-1825.

[6] Niukkanen, A.W., Generalized reduction formulae for multiple hypergeometric series ${ }^{N} F\left(x_{1}, \ldots, x_{n}\right)$, J. Phys. A17 (1984), L16-L31.

[7] Slater, L.J., Generalized Hypergeometric Functions, Cambridge University Press 1966.

[8] Srivastava, H.M., A class of generalized multiple hypergeometric series arising in physical and quantum chemical applications, J. Phys. A18 (1985), L227L234.

[9] Srivastava, H.M., Reduction and summation formulae for a certain class of generalized multiple hypergeometric series arising in physical and quantum chemical applications, J. Phys. A18 (1985), 3079-3085.

[10] Srivastava, H.M., Neumann expansions for a certain class of generalized multiple hypergeometric series arising in physical and quantum chemical applications, J. Phys. A20 (1987), 847-855.

[11] Srivastava, H.M., Some Clebsch-Gordon type linearization relations and other polynomial expansions associated with a class of generalized multiple hypergeometric series arising in physical and quantum chemical applications, $J$. Phys. A21 (1988), 4463-4470.

[12] Srivastava, H.M. and Karlsson, P.W., Multiple Gaussian Hypergeometric Series, Halsted Press, New York 1985. 


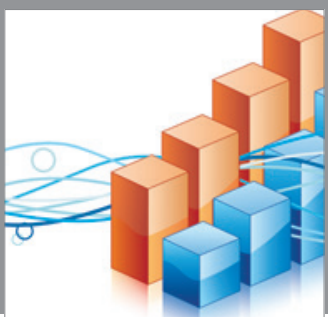

Advances in

Operations Research

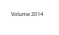

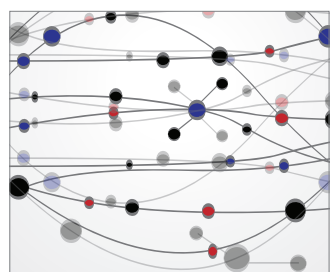

\section{The Scientific} World Journal
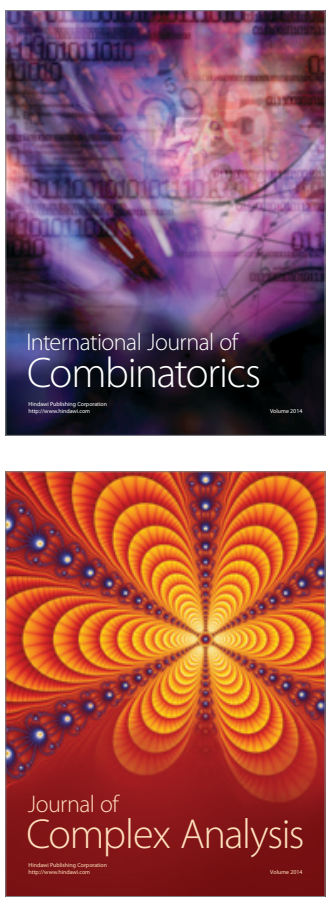

International Journal of

Mathematics and

Mathematical

Sciences
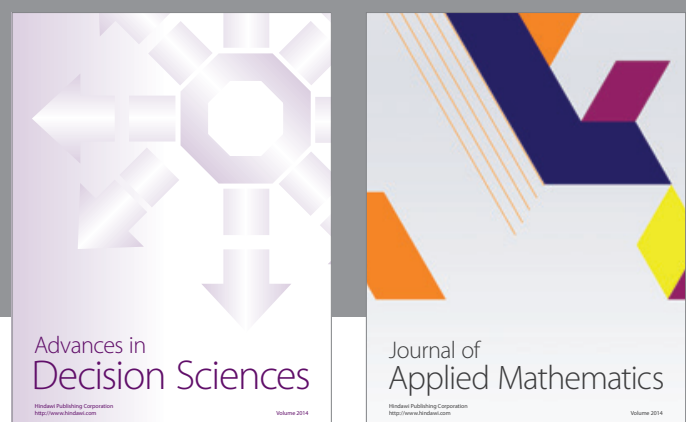

Journal of

Applied Mathematics
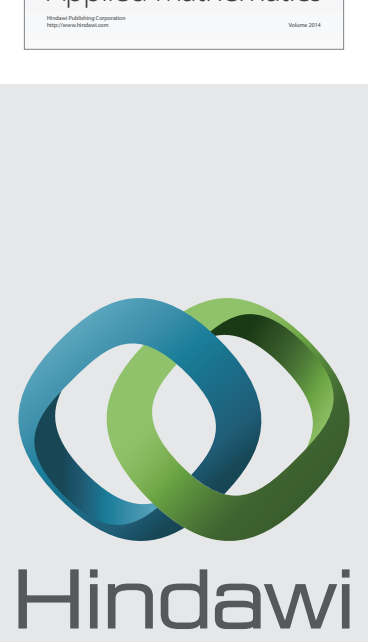

Submit your manuscripts at http://www.hindawi.com
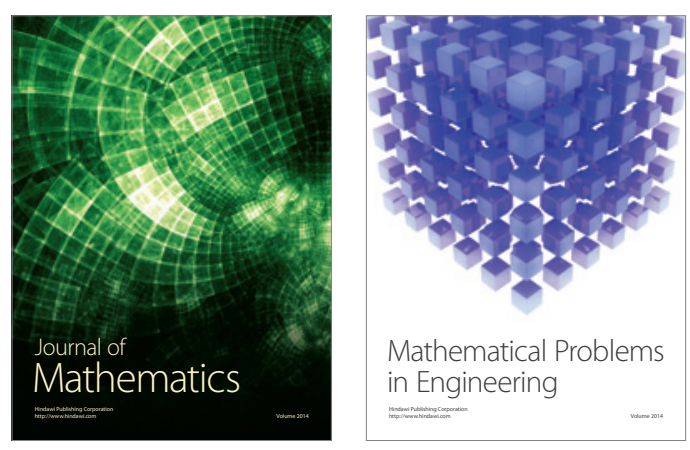

Mathematical Problems in Engineering
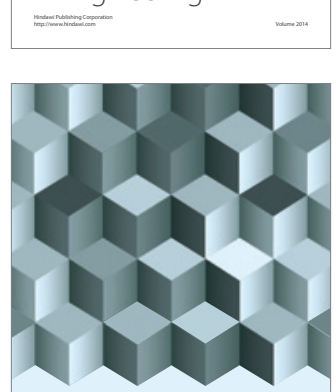

Journal of

Function Spaces
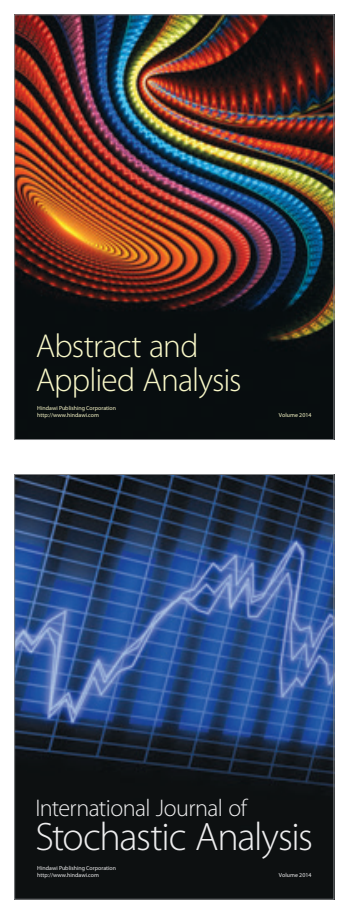

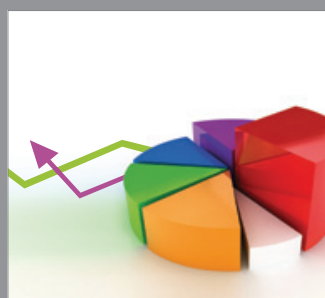

ournal of

Probability and Statistics

Promensencen
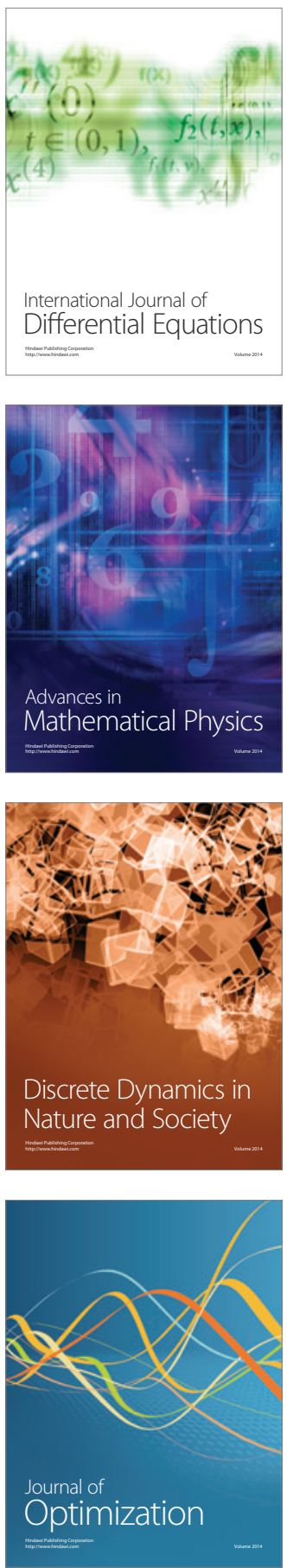